\title{
Fixed bin frequency distribution for the VNTR Loci D2S44, D4S139, D5S110, and D8S358 in a population sample from Minas Gerais, Brazil
}

\author{
Kleber Simônio Parreira ${ }^{1}$, Gismar Silva Vieira ${ }^{2}$, Juarez Inácio ${ }^{2}$, Rodrigo Soares Moura-Neto ${ }^{3}$ \\ and Luiz Ricardo Goulart ${ }^{1}$ \\ ${ }^{1}$ Instituto de Genética e Bioquímica, UFU, Uberlândia, MG, Brazil. \\ ${ }^{2}$ BioGenetics Tecnologia Molecular, Uberlândia, MG, Brazil. \\ ${ }^{3}$ Departamento de Genética, Instituto de Biologia, UFRJ, Rio de Janeiro, RJ, Brazil.
}

\begin{abstract}
Fixed bin frequencies for the VNTR loci D2S44, D4S139, D5S110, and D8S358 were determined in a Minas Gerais population sample. The data were generated by RFLP analysis of Haelll-digested genomic DNA and chemiluminescent detection. The four VNTR loci have met Hardy-Weinberg equilibrium, and there was no association of alleles among VNTR loci. The frequency data can be used in forensic analyses and paternity tests to estimate the frequency of a DNA profile in the general Brazilian population.
\end{abstract}

Key words: fixed-bin frequency, VNTR loci, Brazilian population.

Received: September 29, 2000; accepted: July 23, 2002.

\section{Introduction}

DNA polymorphisms detection through restriction fragment length polymorphism (RFLP) analysis has been used as a powerful tool for identity testing in forensic science (Budowle and Baechtel, 1990). Individuals are distinguished by VNTR (variable number of tandem repeats) variation, and their frequency distribution may be classified into a small number of fixed bins, a common technical approach that allows genotyping unambiguously. However, to perform identity tests through VNTRs, a database of fixed bin frequency is required. This investigation describes the RFLP allelic frequencies of the Minas Gerais population, Brazil, distributed in fixed bins for the HaeIIIgenerated VNTR loci D2S44, D4S139, D5S110, and D8S358.

\section{Materials and Methods}

The source of DNA consisted of blood samples collected randomly from both unrelated parents of trios that underwent paternity tests. These samples were chosen from a blood bank of a Private Reference Laboratory of Minas Gerais State, which were collected in a three-year period (1997 to 1999). Samples were taken from White and Mulatto individuals; however, due to the great racial mixture in Brazil, ethnic composition was not properly annotated, but

Send correspondence to Luiz R. Goulart. Instituto de Genética e Bioquímica, UFU, Uberlândia, MG, Brazil. E-mail: Irgoulart@ufu.br. mulattoes represented the majority of the sample $(80 \%)$. DNA was extracted organically from peripheral blood samples, digested with HaeIII, and separated in $0.8 \%$ agarose gel electrophoresis. Southern transferred to nylon membrane (Byodine A) were hybridized to alkaline phosphatase-conjugated probes (ACES 2.0 plus Kit), according to the supplier's protocol (Life Technologies, Gibco-BRL, Gaithersburg, MD). The probes PH30 (D4S139), LH1 (D5S110), and CEB42 (D8S358) were purchased from Life Technologies (Gibco-BRL, Gaithersburg, MD). The probe YNH4 (D2S44) probe was supplied by LifeCodes Corp. (Valhalla, NY). The estimated base pair sizing of the digested DNA was performed based on a Gibco-BRL molecular weight marker (i.e. sizing ladder) in a video documentation system (ImageMaster VDS System, Pharmacia Biotech, San Francisco, CA). The allele size data were distributed in fixed bins according to Budowle et al. (1991a).

Hardy-Weinberg equilibrium (HWE) was demonstrated by the likelihood ratio test (Edwards et al., 1992) and by the exact test (Guo and Thompson, 1992). An interclass correlation criterion for two-locus associations was used to detect disequilibrium between VNTR loci (Karlin et al., 1981). Independence across the four VNTR loci was determined by examining whether the observed variance of the number of heterozygous loci in the population sample was outside its confidence interval under the assumption of independence (Chakraborty, 1984). 


\section{Results and Discussion}

Table I presents the 31 fixed bin frequency distributions for the four highly polymorphic VNTR loci in the Minas Gerais population. This is the first report on the distribution of bin frequencies for the D8S358 VNTR locus in the general Brazilian population. Statistical analysis demonstrated that allelic frequencies at all four loci are in HW equilibrium, and there was no correlation among alleles in any of the loci pairs based on the interclass correlation tests. Furthermore, there was no evidence of association among the four loci described in this population sample using the $\mathrm{S}_{\mathrm{k}}{ }^{2}$ criterion $\left(\mathrm{S}_{\mathrm{k}}{ }^{2}=0.302\right.$, confidence interval 0.162-0.435).

Population data (bins) of the four VNTR loci for Caucasians in the United States (Budowle et al., 1994) were compared to our Brazilian data. There were very few instances in which substantial differences in fixed bin frequencies could be observed between the two population samples, even considering the great difference of in ethnical composition of both countries. Under the assumption of independence, this result suggests that there would

Table I - Fixed bin frequencies for four VNTR loci in Minas Gerais, Brazil.

\begin{tabular}{|c|c|c|c|c|c|}
\hline Bin & Size (bp) & D2S44 & D4S139 & D5S110 & D8S358 \\
\hline 1 & $1-639$ & 0.000 & 0.000 & 0.000 & 0.002 \\
\hline 2 & $640-772$ & 0.019 & 0.000 & 0.002 & 0.005 \\
\hline 3 & $773-871$ & 0.004 & 0.000 & 0.002 & 0.000 \\
\hline 4 & $872-963$ & 0.016 & 0.000 & 0.002 & 0.000 \\
\hline 5 & $964-1077$ & 0.008 & 0.000 & 0.004 & 0.000 \\
\hline 6 & $1078-1196$ & 0.027 & 0.000 & 0.005 & 0.009 \\
\hline 7 & $1197-1352$ & 0.086 & 0.000 & 0.007 & 0.023 \\
\hline 8 & $1353-1507$ & 0.057 & 0.000 & 0.030 & 0.040 \\
\hline 9 & $1508-1637$ & 0.105 & 0.002 & 0.021 & 0.023 \\
\hline 10 & $1638-1788$ & 0.103 & 0.000 & 0.030 & 0.028 \\
\hline 11 & $1789-1924$ & 0.055 & 0.000 & 0.027 & 0.019 \\
\hline 12 & $1925-2088$ & 0.047 & 0.000 & 0.047 & 0.042 \\
\hline 13 & $2089-2351$ & 0.076 & 0.010 & 0.086 & 0.053 \\
\hline 14 & $2352-2522$ & 0.035 & 0.000 & 0.056 & 0.035 \\
\hline 15 & $2523-2692$ & 0.033 & 0.010 & 0.066 & 0.065 \\
\hline 16 & $2693-2862$ & 0.074 & 0.002 & 0.066 & 0.070 \\
\hline 17 & $2863-3033$ & 0.078 & 0.002 & 0.059 & 0.084 \\
\hline 18 & $3034-3329$ & 0.057 & 0.020 & 0.079 & 0.081 \\
\hline 19 & $3330-3674$ & 0.053 & 0.033 & 0.099 & 0.105 \\
\hline 20 & $3675-3979$ & 0.027 & 0.037 & 0.054 & 0.056 \\
\hline 21 & $3980-4323$ & 0.018 & 0.047 & 0.045 & 0.056 \\
\hline 22 & $4324-4821$ & 0.002 & 0.084 & 0.072 & 0.044 \\
\hline 23 & $4822-5219$ & 0.002 & 0.069 & 0.018 & 0.030 \\
\hline 24 & $5220-5685$ & 0.006 & 0.074 & 0.047 & 0.040 \\
\hline 25 & $5686-6368$ & 0.008 & 0.141 & 0.020 & 0.049 \\
\hline 26 & $6369-7241$ & 0.004 & 0.166 & 0.023 & 0.028 \\
\hline 27 & $7242-8452$ & 0.000 & 0.111 & 0.023 & 0.005 \\
\hline 28 & $8453-10093$ & 0.000 & 0.097 & 0.009 & 0.007 \\
\hline 29 & $10094-11368$ & 0.000 & 0.027 & 0.000 & 0.000 \\
\hline 30 & $11369-12829$ & 0.000 & 0.022 & 0.000 & 0.002 \\
\hline 31 & $12830-25000$ & 0.000 & 0.047 & 0.000 & 0.000 \\
\hline Number of chromosomes & & 512 & 596 & 558 & 430 \\
\hline Individuals-single band & & 16 & 23 & 22 & 15 \\
\hline HWE/Likelihood ratio (p =) & & 0.866 & 0.081 & 0.127 & 0.167 \\
\hline HWE/Exact test $(\mathrm{p}=)$ & & 0.742 & 0.093 & 0.148 & 0.141 \\
\hline
\end{tabular}


be little difference in multiple loci profile frequency estimates using both databases.

In conclusion, this article provides data for estimated frequencies in fixed bins of D2S44, D4S139, D5S110 and D8S358 VNTR loci. Results demonstrated that the fixed bin frequencies of the four loci can be used in forensic analysis and paternity tests for the Brazilian population.

\section{Acknowledgments}

Sampling and paternity tests were performed at the BioGenetics Tecnologia Molecular Laboratory, Minas Gerais, Brazil. Research fellowship supported by CNPq.

\section{References}

Budowle B and Baechtel FS (1990) Modifications to improve the effectiveness of restriction fragment length polymorphism typing. Appl. Theor. Electrophoresis 1:181-187.
Budowle B, Giusti AM, Waye JS, Baechtel FS, Foumey RM, Adams de, Presley LA, Deadman HA and Monson KL (1991a) Fixed-bin analysis for statistical evaluation of continuous distributions of allelic data from VNTR loci, for use in forensic comparisons. Am J Hum Genet 48:841-855.

Budowle B, Monson KL, Giusti AM, Brown BL (1994) The assessment of frequency estimates of Hae III-generated VNTR profiles in various reference databases. J Forensic Sci 39(2):319-52.

Chakraborty R (1984) Detection of nonrandom association of alleles from the distribution of the number of heterozygous loci in a sample. Genetics 108:719-731.

Edwards A, Hammond H, Jin L, Caskey CR and Chakraborty R (1992) Genetic variation at five trimeric and tetrameric repeat loci in four human population groups. Genomics 12:241-253.

Guo SW and Thompson EA (1992) Performing the exact test of Hardy-Weinberg proportion for multiple alleles. Biometrics 48:361-372.

Karlin S, Cameron EC and Williams PT (1981) Sibling and parent-offspring correlation estimation with variable family size. Proc Nat Acad Sci USA 78:2664-8. 